Eur. J. Math. Anal. 1 (2021) 34-44

doi: 10.28924/ada/ma.1.34

\title{
Katugampola Fractional Calculus With Generalized k-Wright Function
}

\author{
Ahmad Y. A. Salamooni ${ }^{1, *}$, D. D. Pawar ${ }^{2}$ \\ ${ }^{1}$ Department of Mathematics, Faculty of Education Zabid, Hodeidah University, Al-Hodeidah, Yemen \\ ayousss83@gmail.com \\ ${ }^{2}$ School of Mathematical Sciences, Swami Ramanand Teerth Marathwada University, Nanded-431606, \\ India \\ dypawar@yahoo.com \\ *Correspondence: ayousss83@gmail.com
}

\begin{abstract}
АвSTRACT. In this article, we present some properties of the Katugampola fractional integrals and derivatives. Also, we study the fractional calculus properties involving Katugampola Fractional integrals and derivatives of generalized $k$-Wright function ${ }_{n} \Phi_{m}^{k}(z)$.
\end{abstract}

\section{Introduction and Preliminaries}

In recent years, researchers have introduced new fractional integral and differential operators which are generalizations of the famous definitions of Riemann-Liouville, Caputo, Hadamard, Hilfer, etc. They have made a qualitative contribution to fractional differential equations. For more details, see $[1,5-7,9-14]$ and references therein.

Definition 1.1. [9] Let $\Omega=[a, b]$, the Katugampola fractional integrals $\rho_{\rho} I_{0+}^{\gamma} \varphi$ and ${ }_{\rho} I_{-}^{\gamma} \varphi$ of order $\gamma \in \mathbb{C}(\Re(\gamma)>0)$ are defined for $\rho>0, a=0$ and $b=\infty$ as

$$
\left({ }_{\rho} \rho_{0+}^{\gamma} \varphi\right)(s)=\frac{\rho^{1-\gamma}}{\Gamma(\gamma)} \int_{0}^{s} \frac{\tau^{\rho-1} \varphi(\tau)}{\left(s^{\rho}-\tau^{\rho}\right)^{1-\gamma}} d \tau \quad(s>0)
$$

and

$$
\left({ }_{\rho} I_{-}^{\gamma} \varphi\right)(s)=\frac{\rho^{1-\gamma}}{\Gamma(\gamma)} \int_{s}^{\infty} \frac{\tau^{\rho-1} \varphi(\tau)}{\left(\tau^{\rho}-s^{\rho}\right)^{1-\gamma}} d \tau \quad(s>0),
$$

the corresponding Katugampola fractional derivatives ${ }_{\rho} D_{0+}^{\gamma} \varphi$ and ${ }_{\rho} D_{-}^{\gamma} \varphi$ are defined with $(n=$ $1+[\Re(\gamma)])$ as

$$
\left({ }_{\rho} D_{0+}^{\gamma} \varphi\right)(s):=\left(s^{1-\rho} \frac{d}{d s}\right)^{1+[\mathfrak{R}(\gamma)]}\left({ }_{\rho} I_{0+}^{1-\gamma+[\mathfrak{R}(\gamma)]} \varphi\right)(s)
$$

Received: 30 Aug 2021.

Key words and phrases. Katugampola fractional integral and derivative; $k$-Gamma function; $k$-Wright function. 


$$
=\frac{\rho^{\gamma-[\mathfrak{R}(\gamma)]}}{\Gamma(1-\gamma+[\mathfrak{R}(\gamma)])}\left(s^{1-\rho} \frac{d}{d s}\right)^{1+[\mathfrak{R}(\gamma)]} \int_{0}^{s} \frac{\tau^{\rho-1} \varphi(\tau)}{\left(s^{\rho}-\tau^{\rho}\right)^{\gamma-[\mathfrak{R}(\gamma)]}} d \tau \quad(s>0),
$$

and

$$
\begin{aligned}
\left({ }_{\rho} D_{-}^{\gamma} \varphi\right)(s) & :=\left(-s^{1-\rho} \frac{d}{d s}\right)^{1+[\Re(\gamma)]}\left({ }_{\rho} I_{-}^{1-\gamma+[\mathfrak{R}(\gamma)]} \varphi\right)(s) \\
& =\frac{\rho^{\gamma-[\mathfrak{R}(\gamma)]}}{\Gamma(1-\gamma+[\mathfrak{R}(\gamma)])}\left(-s^{1-\rho} \frac{d}{d s}\right)^{1+[\mathfrak{R}(\gamma)]} \int_{s}^{\infty} \frac{\tau^{\rho-1} \varphi(\tau)}{\left(\tau^{\rho}-s^{\rho}\right)^{\gamma-[\mathfrak{R}(\gamma)]}} d \tau \quad(s>0) .
\end{aligned}
$$

Definition 1.2. [2] The generalized $K$-Gamma function $\Gamma_{k}(y)$ is defined by

$$
\Gamma_{k}(y)=\lim _{n \rightarrow \infty} \frac{n ! k^{n}(n k)^{\frac{y}{k}-1}}{(y)_{n, k}} \quad\left(k>0 ; y \in \mathbb{C} \backslash k \mathbb{Z}^{-}\right),
$$

where $(y)_{n, k}$ is the $k$-Pochhammer symbol given as

$$
(y)_{n, k}:= \begin{cases}\frac{\Gamma_{k}(y+n k)}{\Gamma_{k}(y)} & (k \in \mathbb{R} ; y \in \mathbb{C} \backslash\{0\}) \\ y(y+k)(y+2 k) \ldots(y+(n-1) k) & \left(n \in \mathbb{N}^{+} ; y \in \mathbb{C}\right)\end{cases}
$$

and for $\mathfrak{R}(y)>0$, the $K$-Gamma function $\Gamma_{k}(y)$ is defined by the integral

$$
\Gamma_{k}(y)=\int_{0}^{\infty} x^{y-1} e^{-\frac{x^{k}}{k}} d x .
$$

This gives a relation with Euler's Gamma function as

$$
\Gamma_{k}(y)=k^{\frac{y}{k}-1} \Gamma\left(\frac{y}{k}\right) \text {. }
$$

Also, in [8], we have

$$
\Gamma(1-y) \Gamma(y)=\frac{\pi}{\sin (y \pi)} .
$$

Definition 1.3. [14] The Beta function $B(v, \omega)$ is defined as

$$
\begin{aligned}
B(v, \omega) & =\int_{0}^{1} z^{v-1}(1-z)^{\omega-1} d z, \quad \mathfrak{R}(v)>0, \quad \mathfrak{R}(\omega)>0, \\
& =\frac{\Gamma(v) \Gamma(\omega)}{\Gamma(v+\omega)}
\end{aligned}
$$

Furthermore, we have

$$
\begin{gathered}
\int_{\hat{x}}^{\infty}(z-\hat{x})^{v-1}(z-\hat{y})^{\omega-1} d z=(\hat{x}-\hat{y})^{v+\omega-1} B(v, 1-v-\omega), \\
\hat{x}>\hat{y}, \quad 0<\mathfrak{R}(v)<1-\mathfrak{R}(\omega) .
\end{gathered}
$$

Recently, the Generalized $K$-Wright function introduced by (Gehlot and Prajapati [3]) is defined as follows: 
Definition 1.4. For $k \in \mathbb{R}^{+} ; \quad z \in \mathbb{C} ; p_{i}, q_{j} \in \mathbb{C}, \alpha_{i}, \beta_{j} \in \mathbb{R}\left(\alpha_{i}, \beta_{j} \neq 0 ; i=1,2, \ldots, n ; j=\right.$ $1,2, \ldots, m)$ and $\left(p_{i}+\alpha_{i} r\right),\left(q_{j}+\beta_{j} r\right) \in \mathbb{C} \backslash k \mathbb{Z}^{-}$, the generalized $k$-Wright function ${ }_{n} \Phi_{m}^{k}$ is defined by

$$
\left.{ }_{n} \Phi_{m}^{k}(z)={ }_{n} \Phi_{m}^{k}\left[\begin{array}{l}
\left(p_{i}, \alpha_{i}\right)_{1, n} \\
\left(q_{j}, \beta_{j}\right)_{1, m}
\end{array}\right] z\right]=\sum_{r=0}^{\infty} \frac{\prod_{i=1}^{n} \Gamma_{k}\left(p_{i}+\alpha_{i} r\right)}{\prod_{j=1}^{m} \Gamma_{k}\left(q_{j}+\beta_{j} r\right)} \frac{z^{r}}{r !},
$$

with the convergence conditions described as

$$
\Delta=\sum_{j=1}^{m}\left(\frac{\beta_{j}}{k}\right)-\sum_{i=1}^{n}\left(\frac{\alpha_{i}}{k}\right) ; \mu=\prod_{i=1}^{n}\left|\frac{\alpha_{i}}{k}\right|^{-\frac{\alpha_{i}}{k}} \prod_{j=1}^{m}\left|\frac{\beta_{j}}{k}\right|^{\frac{\beta_{j}}{k}} ; \nu=\sum_{j=1}^{m}\left(\frac{q_{j}}{k}\right)-\sum_{i=1}^{n}\left(\frac{p_{i}}{k}\right)+\frac{n-m}{2} .
$$

Lemma 1.1. [3] For $k \in \mathbb{R}^{+} ; \quad z \in \mathbb{C} ; p_{i}, q_{j} \in \mathbb{C}, \alpha_{i}, \beta_{j} \in \mathbb{R}\left(\alpha_{i}, \beta_{j} \neq 0 ; i=1,2, \ldots, n ; j=\right.$ $1,2, \ldots, m)$ and $\left(p_{i}+\alpha_{i} r\right),\left(q_{j}+\beta_{j} r\right) \in \mathbb{C} \backslash k \mathbb{Z}^{-}$

(1) If $\Delta>-1$, then series (1.12) is absolutely convergent for all $z \in \mathbb{C}$ and generalized $k$-Wright function ${ }_{n} \Phi_{m}^{k}(z)$ is an entire function of $z$.

(2) If $\Delta=-1$, then series (1.12) is absolutely convergent for all $|z|<\mu$ and of

$$
|z|=\mu, \mathfrak{R}(\mu)>\frac{1}{2} .
$$

\section{Properties of Katugampola Fractional Integral and Derivative}

In this section, we investigate some properties of the Katugampola fractional integrals and derivatives (1.1), (1.2) and (1.3), (1.4) for the power function $\varphi(s)=s^{\alpha-1}$ and the exponential function $e^{-\lambda s^{\rho}}$.

Lemma 2.1. Let $\rho>0, \mathfrak{R}(\gamma) \geqq 0$ and $n=1+[\mathfrak{R}(\gamma)]$

(1) If $\mathfrak{R}(\alpha)>0$, then

$$
\begin{aligned}
& \left({ }_{\rho} I_{0+}^{\gamma} \tau^{\alpha-1}\right)(s)=\frac{\rho^{-\gamma} \Gamma\left(1+\frac{\alpha-1}{\rho}\right)}{\Gamma\left(1+\frac{\alpha-1}{\rho}+\gamma\right)} s^{\rho \gamma+(\alpha-1)} \quad(\mathfrak{R}(\gamma) \geq 0 ; \mathfrak{R}(\alpha)>0) \\
& \left({ }_{\rho} D_{0+}^{\gamma} \tau^{\alpha-1}\right)(s)=\frac{\rho^{\gamma-n} \Gamma\left(1+\frac{\alpha-1}{\rho}\right)}{\Gamma\left(1+\frac{\alpha-1}{\rho}-\gamma\right)} s^{(\alpha-1)-\rho \gamma} \quad(\mathfrak{R}(\gamma) \geqq 0 ; \mathfrak{R}(\alpha)>0) .
\end{aligned}
$$

(2) If $\alpha \in \mathbb{C}$, then

$$
\begin{gathered}
\left({ }_{\rho} I_{-}^{\gamma} \tau^{\alpha-1}\right)(s)=\frac{\rho^{-\gamma} \Gamma\left(\frac{1-\alpha}{\rho}-\gamma\right)}{\Gamma\left(\frac{1-\alpha}{\rho}\right)} s^{\rho \gamma+(\alpha-1)} \quad(\mathfrak{R}(\gamma) \geq 0 ; \mathfrak{R}(\gamma+\alpha)<1) \\
\left({ }_{\rho} D_{-}^{\gamma} \tau^{\alpha-1}\right)(s)=\frac{\rho^{\gamma-n} \Gamma\left(\frac{1-\alpha}{\rho}+\gamma\right)}{\Gamma\left(\frac{1-\alpha}{\rho}\right)} s^{(\alpha-1)-\rho \gamma} \quad(\mathfrak{R}(\gamma) \geqq 0 ; \mathfrak{R}(\gamma+\alpha-[\mathfrak{R}(\gamma)])<1) .
\end{gathered}
$$

(3) If $\mathfrak{R}(\lambda)>0$, then

$$
\left({ }_{\rho} I_{-}^{\gamma} e^{-\lambda \tau^{\rho}}\right)(s)=(\lambda \rho)^{-\gamma} e^{-\lambda s^{\rho}} \quad(\Re(\gamma) \geq 0)
$$




$$
\left({ }_{\rho} D_{-}^{\gamma} e^{-\lambda \tau^{\rho}}\right)(s)=(\lambda \rho)^{\gamma} e^{-\lambda s^{\rho}} \quad(\Re(\gamma) \geqq 0) .
$$

Proof. To prove this Lemma, let the substitution $x=\frac{\tau^{\rho}}{s^{\rho}}$ in parts (1) and (2).

(1) Firstly, by the equation (1.1) and the given substitution, we have

$$
\begin{aligned}
\left({ }_{\rho} l_{0+}^{\gamma} \tau^{\alpha-1}\right)(s) & =\frac{\rho^{-\gamma} S^{\rho \gamma+\alpha-1}}{\Gamma(\gamma)} \int_{0}^{1} \frac{x^{\frac{\alpha-1}{\rho}}}{(1-x)^{1-\gamma}} d x \\
& =\frac{\rho^{-\gamma} S^{\rho \gamma+\alpha-1}}{\Gamma(\gamma)} B\left(\gamma, 1+\frac{\alpha-1}{\rho}\right) .
\end{aligned}
$$

Now, using equation (1.10), we obtain the result (2.1).

Secondly, by the equation (1.3), the given substitution and by using the result (2.1), we have

$$
\begin{aligned}
\left({ }_{\rho} D_{0+}^{\gamma} \tau^{\alpha-1}\right)(s) & =\left(s^{1-\rho} \frac{d}{d s}\right)^{n}\left({ }_{\rho} I_{0+}^{n-\gamma} \tau^{\alpha-1}\right)(s) \\
& =\frac{\rho^{\gamma-n} \Gamma\left(1+\frac{\alpha-1}{\rho}\right)}{\Gamma\left(1+\frac{\alpha-1}{\rho}+n-\gamma\right)}\left(s^{1-\rho} \frac{d}{d s}\right)^{n} s^{\rho(n-\gamma)+\alpha-1} \\
& =\frac{\rho^{\gamma-n} \Gamma\left(1+\frac{\alpha-1}{\rho}\right)}{\Gamma\left(1+\frac{\alpha-1}{\rho}-\gamma\right)} s^{(\alpha-1)-\rho \gamma} .
\end{aligned}
$$

(2) Firstly, by the equation (1.2) and the given substitution, we have

$$
\left({ }_{\rho} I_{-}^{\gamma} \tau^{\alpha-1}\right)(s)=\frac{\rho^{-\gamma} S^{\rho \gamma+\alpha-1}}{\Gamma(\gamma)} \int_{1}^{\infty} x^{\frac{\alpha-1}{\rho}}(x-1)^{\gamma-1} d x .
$$

Now, using the equation (1.11) with $\hat{x}=1$ and $\hat{y}=0$, we obtain

$$
\left({ }_{\rho} I_{-}^{\gamma} \tau^{\alpha-1}\right)(s)=\frac{\rho^{-\gamma}{ }_{s}^{\rho \gamma+\alpha-1}}{\Gamma(\gamma)} B\left(\gamma, 1-\gamma-\left(1+\frac{\alpha-1}{\rho}\right)\right) .
$$

By using equation (1.10), we obtain the result (2.3).

Secondly, by the equation (1.4), the given substitution and by using the result (2.3), we have

$$
\begin{aligned}
\left({ }_{\rho} D_{-}^{\gamma} \tau^{\alpha-1}\right)(s) & =\left(-s^{1-\rho} \frac{d}{d s}\right)^{n}\left({ }_{\rho} I_{-}^{n-\gamma} \tau^{\alpha-1}\right)(s) \\
& =\frac{(-1)^{n} \rho^{\gamma-n} \Gamma\left(\frac{1-\alpha}{\rho}+\gamma-n\right)}{\Gamma\left(\frac{1-\alpha}{\rho}\right)}\left(s^{1-\rho} \frac{d}{d s}\right)^{n} s^{\rho(n-\gamma)+\alpha-1} \\
& =\frac{(-1)^{n} \rho^{\gamma-n}}{\Gamma\left(\frac{1-\alpha}{\rho}\right)} \frac{\Gamma\left(\frac{1-\alpha}{\rho}+\gamma-n\right) \Gamma\left(1-\left[\frac{1-\alpha}{\rho}+\gamma-n\right]\right)}{\Gamma\left(1-\left[\gamma-\frac{\alpha-1}{\rho}\right]\right)} .
\end{aligned}
$$

Also, by using (1.9), we have

$$
\begin{aligned}
& \Gamma\left(\frac{1-\alpha}{\rho}+\gamma-n\right) \Gamma\left(1-\left[\frac{1-\alpha}{\rho}+\gamma-n\right]\right)=\frac{\pi}{\sin \left(\left[\frac{1-\alpha}{\rho}+\gamma-n\right] \pi\right)}=\frac{(-1)^{n} \pi}{\sin \left(\left[\gamma-\frac{\alpha-1}{\rho}\right] \pi\right)} \\
& \text { and } \\
& \frac{1}{\Gamma\left(1-\left[\gamma-\frac{\alpha-1}{\rho}\right]\right)}=\frac{\Gamma\left(\gamma-\frac{\alpha-1}{\rho}\right)}{\Gamma\left(\gamma-\frac{\alpha-1}{\rho}\right) \Gamma\left(1-\left[\gamma-\frac{\alpha-1}{\rho}\right]\right)}=\frac{\Gamma\left(\gamma-\frac{\alpha-1}{\rho}\right)}{\pi} \sin \left(\left[\gamma-\frac{\alpha-1}{\rho}\right] \pi\right)
\end{aligned}
$$

Substituting relations (2.8) and (2.9) in (2.7), we obtain (2.4). 
(3) For this part, let the substitution $x=\tau^{\rho}-s^{\rho}$.

Firstly, by the equation (1.2) and the given substitution in this part, we have

$$
\left({ }_{\rho} I_{-}^{\gamma} e^{-\lambda \tau^{\rho}}\right)(s)=\frac{\rho^{-\gamma}}{\Gamma(\gamma)} e^{-\lambda s^{\rho}} \int_{0}^{\infty} e^{-\lambda x} x^{\gamma-1} d x
$$

then by use the substitution $\vartheta=\lambda x$, we obtain

$$
\left({ }_{\rho} I_{-}^{\gamma} e^{-\lambda \tau^{\rho}}\right)(s)=\frac{\rho^{-\gamma}}{\Gamma(\gamma)} e^{-\lambda s^{\rho}} \lambda^{-\gamma} \int_{0}^{\infty} e^{-\vartheta} \vartheta^{\gamma-1} d \vartheta
$$

since $\int_{0}^{\infty} e^{-\vartheta} \vartheta^{\gamma-1} d \vartheta=\Gamma(\gamma)[8]$, then the result is satisfied.

Secondly, by the equation (1.4) and by using the result (2.5), we have

$$
\begin{aligned}
\left({ }_{\rho} D_{-}^{\gamma} e^{-\lambda \tau^{\rho}}\right)(s) & =\left(-s^{1-\rho} \frac{d}{d s}\right)^{n}\left(\rho_{\rho} I_{-}^{n-\gamma} e^{-\lambda \tau^{\rho}}\right)(s) \\
& =(-1)^{n}\left(s^{1-\rho} \frac{d}{d s}\right)^{n}\left((\lambda \rho)^{\gamma-n} e^{-\lambda s^{\rho}}\right) \\
& =(-1)^{n} s^{(1-\rho) n}(\lambda \rho)^{\gamma-n}\left(\frac{d^{n}}{d s^{n}} e^{-\lambda s^{\rho}}\right) \\
& =(\lambda \rho)^{\gamma} e^{-\lambda s^{\rho}} .
\end{aligned}
$$

Remark 2.1. (a) In Lemma 2.1, if the power function is $\varphi(s)=\left(\frac{s^{\rho}}{\rho}\right)^{\alpha-1}$, then (1) If $\mathfrak{R}(\alpha)>0$, then

$$
\begin{gathered}
\left({ }_{\rho} I_{0+}^{\gamma}\left(\frac{\tau^{\rho}}{\rho}\right)^{\alpha-1}\right)(s)=\frac{\Gamma(\alpha)}{\Gamma(\alpha+\gamma)}\left(\frac{s^{\rho}}{\rho}\right)^{\alpha+\gamma-1} \quad(\mathfrak{R}(\gamma) \geq 0 ; \mathfrak{R}(\alpha)>0) \\
\left({ }_{\rho} D_{0+}^{\gamma}\left(\frac{\tau^{\rho}}{\rho}\right)^{\alpha-1}\right)(s)=\frac{\Gamma(\alpha)}{\Gamma(\alpha-\gamma)}\left(\frac{s^{\rho}}{\rho}\right)^{\alpha-\gamma-1} \quad(\mathfrak{R}(\gamma) \geqq 0 ; \mathfrak{R}(\alpha)>0) .
\end{gathered}
$$

(2) If $\alpha \in \mathbb{C}$, then

$$
\begin{gathered}
\left(\rho_{-}^{\gamma}\left(\frac{\tau^{\rho}}{\rho}\right)^{\alpha-1}\right)(s)=\frac{\Gamma(1-\gamma-\alpha)}{\Gamma(1-\alpha)}\left(\frac{s^{\rho}}{\rho}\right)^{\alpha+\gamma-1} \quad(\mathfrak{R}(\gamma) \geq 0 ; \mathfrak{R}(\gamma+\alpha)<1) \\
\left({ }_{\rho} D_{-}^{\gamma}\left(\frac{\tau^{\rho}}{\rho}\right)^{\alpha-1}\right)(s)=\frac{\Gamma(1+\gamma-\alpha)}{\Gamma(1-\alpha)}\left(\frac{s^{\rho}}{\rho}\right)^{\alpha-\gamma-1} \quad(\mathfrak{R}(\gamma) \geqq 0 ; \mathfrak{R}(\gamma+\alpha-[\mathfrak{R}(\gamma)])<1) .
\end{gathered}
$$

(b) If $\mathfrak{R}(\alpha)>\mathfrak{R}(\gamma)>0$, then

$$
\left({ }_{\rho} I_{-}^{\gamma} \tau^{-\alpha}\right)(s)=\frac{\rho^{-\gamma} \Gamma\left(\frac{\alpha}{\rho}-\gamma\right)}{\Gamma\left(\frac{\alpha}{\rho}\right)} s^{\rho \gamma-\alpha} .
$$




\section{Katugampola Fractional integration for Generalized $k$-Wright Function}

In this section, we establish the Katugampola fractional integration for generalized $k$-Wright function (1.12).

Theorem 3.1. Let $\gamma, \alpha \in \mathbb{C}$ such that $\mathfrak{R}(\gamma)>0, \mathfrak{R}(\alpha)>0 ; \lambda \in \mathbb{C}, \rho>0, \nu>0$, then for $\Delta>-1$, the Katugampola fractional integration $\rho_{\rho}^{\gamma}{ }_{0+}^{\gamma}$ for generalized $k$-Wright function ${ }_{n} \Phi_{m}^{k}(z)$ is given as

$$
\begin{aligned}
& \left(\rho I_{0+}^{\gamma}\left(\tau_{n}^{\frac{\alpha}{k}-1} \Phi_{m}^{k}\left[\begin{array}{l}
\left(p_{i}, \alpha_{i}\right)_{1, n} \\
\left(q_{j}, \beta_{j}\right)_{1, m}
\end{array} \mid \lambda \tau^{\frac{\nu}{k}}\right]\right)\right)(s) \\
& \quad=\left(\frac{k}{\rho}\right)^{\gamma} s^{\frac{\alpha}{k}+\rho \gamma-1}{ }_{n+1} \Phi_{m+1}^{k}\left[\begin{array}{c}
\left(p_{i}, \alpha_{i}\right)_{1, n},\left(\frac{1}{\rho}(\alpha+(\rho-1) k), \frac{\nu}{\rho}\right) \\
\left(q_{j}, \beta_{j}\right)_{1, m},\left(\frac{1}{\rho}(\alpha+(\rho(\gamma+1)-1) k), \frac{\nu}{\rho}\right)
\end{array} \mid \lambda s^{\frac{\nu}{k}}\right] .
\end{aligned}
$$

Proof. According to Lemma 1.1, a generalized $k$-Wright function in both sides of the equation (3.1) exists for $s>0$. We consider that

$$
M \equiv\left(\rho_{0+}^{\gamma}\left(\tau^{\frac{\alpha}{k}-1}{ }_{n} \Phi_{m}^{k}\left[\begin{array}{c}
\left(p_{i}, \alpha_{i}\right)_{1, n} \\
\left(q_{j}, \beta_{j}\right)_{1, m}
\end{array} \mid \lambda \tau^{\frac{\nu}{k}}\right]\right)\right)(s) .
$$

Using (1.12), we can write the above equation as

$$
M \equiv\left(\rho I_{0+}^{\gamma}\left(\tau^{\frac{\alpha}{k}-1} \sum_{r=0}^{\infty} \frac{\prod_{i=1}^{n} \Gamma_{k}\left(p_{i}+\alpha_{i} r\right)}{\prod_{j=1}^{m} \Gamma_{k}\left(q_{j}+\beta_{j} r\right)} \frac{\left(\lambda \tau^{\frac{\nu}{k}}\right)^{r}}{r !}\right)\right)(s) .
$$

Now, using the integration of the series term by term, we obtain

$$
M \equiv \sum_{r=0}^{\infty} \frac{\prod_{i=1}^{n} \Gamma_{k}\left(p_{i}+\alpha_{i} r\right)}{\prod_{j=1}^{m} \Gamma_{k}\left(q_{j}+\beta_{j} r\right)} \frac{(\lambda)^{r}}{r !}\left(\rho_{0+}^{\gamma}\left(\tau^{\frac{\alpha}{k}+\frac{\nu r}{k}-1}\right)\right)(s)
$$

Applying (2.1), the above equation is reduced to

$$
M \equiv \sum_{r=0}^{\infty} \frac{\prod_{i=1}^{n} \Gamma_{k}\left(p_{i}+\alpha_{i} r\right)}{\prod_{j=1}^{m} \Gamma_{k}\left(q_{j}+\beta_{j} r\right)} \frac{(\lambda)^{r}}{r !} \frac{\rho^{-\gamma} \Gamma\left(1+\frac{\frac{\alpha}{k}+\frac{\nu r}{k}-1}{\rho}\right)}{\Gamma\left(1+\frac{\frac{\alpha}{k}+\frac{\nu \nu}{k}-1}{\rho}+\gamma\right)} s^{\frac{\alpha+\nu r}{k}+\rho \gamma-1}
$$

Using (1.8), we obtain

$$
M \equiv\left(\frac{k}{\rho}\right)^{\gamma} s^{\frac{\alpha}{k}+\rho \gamma-1}{ }_{n+1} \Phi_{m+1}^{k}\left[\begin{array}{c}
\left(p_{i}, \alpha_{i}\right)_{1, n},\left(\frac{1}{\rho}(\alpha+(\rho-1) k), \frac{\nu}{\rho}\right) \\
\left(q_{j}, \beta_{j}\right)_{1, m^{\prime}}\left(\frac{1}{\rho}(\alpha+(\rho(\gamma+1)-1) k), \frac{\nu}{\rho}\right)
\end{array} \mid \lambda s^{\frac{\nu}{k}}\right] .
$$

Theorem 3.2. Let $\gamma, \alpha \in \mathbb{C}$ such that $\mathfrak{R}(\gamma)>0, \mathfrak{R}(\alpha)>0 ; \lambda \in \mathbb{C}, \rho>0, \quad \nu>0$, then for $\Delta>-1$, the Katugampola fractional integration $\rho_{-}^{\gamma}$ for generalized $k$-Wright function ${ }_{n} \Phi_{m}^{k}(z)$ is given as

$$
\left(\rho I_{-}^{\gamma}\left(\tau^{-\frac{\alpha}{k}}{ }_{n} \Phi_{m}^{k}\left[\begin{array}{c}
\left(p_{i}, \alpha_{i}\right)_{1, n} \\
\left(q_{j}, \beta_{j}\right)_{1, m}
\end{array} \mid \lambda \tau^{-\frac{\nu}{k}}\right]\right)\right)(s)
$$




$$
=\left(\frac{k}{\rho}\right)^{\gamma} s^{\rho \gamma-\frac{\alpha}{k}}{ }_{n+1} \Phi_{m+1}^{k}\left[\begin{array}{c}
\left(p_{i}, \alpha_{i}\right)_{1, n^{\prime}}\left(\frac{\alpha}{\rho}-k \gamma, \frac{\nu}{\rho}\right) \\
\left(q_{j}, \beta_{j}\right)_{1, m^{\prime}}\left(\frac{\alpha}{\rho}, \frac{\nu}{\rho}\right)
\end{array} \mid \lambda s^{-\frac{\nu}{k}}\right] .
$$

Proof. According to Lemma 1.1, a generalized $k$-Wright function in both sides of the equation (3.2) exists for $s>0$. We consider that

$$
N \equiv\left(\rho I_{-}^{\gamma}\left(\tau^{-\frac{\alpha}{k}}{ }_{n} \Phi_{m}^{k}\left[\begin{array}{c}
\left(p_{i}, \alpha_{i}\right)_{1, n} \\
\left(q_{j}, \beta_{j}\right)_{1, m}
\end{array} \mid \lambda \tau^{-\frac{\nu}{k}}\right]\right)\right)(s) .
$$

Using (1.12), we can write the above equation as

$$
N \equiv \sum_{r=0}^{\infty} \frac{\prod_{i=1}^{n} \Gamma_{k}\left(p_{i}+\alpha_{i} r\right)}{\prod_{j=1}^{m} \Gamma_{k}\left(q_{j}+\beta_{j} r\right)} \frac{(\lambda)^{r}}{r !}\left(\rho I_{-}^{\gamma}\left(\tau^{-\frac{\alpha+\nu r}{k}}\right)\right)(s) .
$$

Applying (2.10), the above equation is reduced to

$$
N \equiv \sum_{r=0}^{\infty} \frac{\prod_{i=1}^{n} \Gamma_{k}\left(p_{i}+\alpha_{i} r\right)}{\prod_{j=1}^{m} \Gamma_{k}\left(q_{j}+\beta_{j} r\right)} \frac{(\lambda)^{r}}{r !} \frac{\rho^{-\gamma} \Gamma\left(\frac{\frac{\alpha+\nu r}{k}}{\rho}-\gamma\right)}{\Gamma\left(\frac{\frac{\alpha+\nu r}{k}}{\rho}\right)} s^{\rho \gamma-\frac{\alpha+\nu r}{k}} .
$$

Using (1.8), we obtain

$$
N \equiv\left(\frac{k}{\rho}\right)^{\gamma} s^{\rho \gamma-\frac{\alpha}{k}}{ }_{n+1} \Phi_{m+1}^{k}\left[\begin{array}{c}
\left(p_{i}, \alpha_{i}\right)_{1, n^{\prime}}\left(\frac{\alpha}{\rho}-k \gamma, \frac{\nu}{\rho}\right) \\
\left(q_{j}, \beta_{j}\right)_{1, m^{\prime}}\left(\frac{\alpha}{\rho}, \frac{\nu}{\rho}\right)
\end{array} \mid \lambda s^{-\frac{\nu}{k}}\right] .
$$

\section{Katugampola Fractional Differentiation for Generalized $k$-Wright Function}

This section deals with the Katugampola fractional differentiation for generalized $k$-Wright function (1.12).

Theorem 4.1. Let $\gamma, \alpha \in \mathbb{C}$ such that $\mathfrak{R}(\gamma)>0, \mathfrak{R}(\alpha)>0 ; \lambda \in \mathbb{C}, \rho>0, \nu>0$, then for $\Delta>-1$, the Katugampola fractional differentiation ${ }_{\rho} D_{0+}^{\gamma}$ for generalized $k$-Wright function ${ }_{n} \Phi_{m}^{k}(z)$ is given as

$$
\begin{aligned}
& \left.\qquad{ }_{\rho} D_{0+}^{\gamma}\left(\tau^{\frac{\alpha}{k}-1}{ }_{n} \Phi_{m}^{k}\left[\begin{array}{l}
\left(p_{i}, \alpha_{i}\right)_{1, n} \\
\left(q_{j}, \beta_{j}\right)_{1, m}
\end{array} \mid \lambda \tau^{\frac{\nu}{k}}\right]\right)\right)(s) \\
& \quad=\left(\frac{k}{\rho}\right)^{-\gamma} s^{\frac{\alpha}{k}-\rho \gamma-1}{ }_{n+1} \Phi_{m+1}^{k}\left[\begin{array}{c}
\left(p_{i}, \alpha_{i}\right)_{1, n},\left(\frac{1}{\rho}(\alpha+(\rho-1) k), \frac{\nu}{\rho}\right) \\
\left(q_{j}, \beta_{j}\right)_{1, m},\left(\frac{1}{\rho}(\alpha+(\rho(1-\gamma)-1) k), \frac{\nu}{\rho}\right)
\end{array} \mid \lambda s^{\frac{\nu}{k}}\right] .
\end{aligned}
$$

Proof. According to Lemma 1.1, a generalized $k$-Wright function in both sides of the equation (4.1) exists for $s>0$. Let $n=1+[\Re(\gamma)]$. Then, we consider that

$$
P \equiv\left({ }_{\rho} D_{0+}^{\gamma}\left(\tau^{\frac{\alpha}{k}-1}{ }_{n} \Phi_{m}^{k}\left[\begin{array}{c}
\left(p_{i}, \alpha_{i}\right)_{1, n} \\
\left(q_{j}, \beta_{j}\right)_{1, m}
\end{array} \mid \lambda \tau^{\frac{\nu}{k}}\right]\right)\right)(s) .
$$


Using (1.3), we have

$$
P \equiv\left(s^{1-\rho} \frac{d}{d s}\right)^{n}\left(\rho_{0+}^{n-\gamma}\left(\tau_{n}^{\frac{\alpha}{k}-1}{ }_{n}^{k}\left[\begin{array}{c}
\left(p_{i}, \alpha_{i}\right)_{1, n} \mid \\
\left(q_{j}, \beta_{j}\right)_{1, m}
\end{array} \mid \lambda \tau^{\frac{\nu}{k}}\right]\right)\right)(s) .
$$

Using Theorem 3.1, we obtain

$$
P \equiv\left(s^{1-\rho} \frac{d}{d s}\right)^{n}\left(\left(\frac{k}{\rho}\right)^{n-\gamma} s^{\frac{\alpha}{k}+\rho(n-\gamma)-1}{ }_{n+1} \Phi_{m+1}^{k}\left[\begin{array}{c}
\left(p_{i}, \alpha_{i}\right)_{1, n^{\prime}}\left(\frac{1}{\rho}(\alpha+(\rho-1) k), \frac{\nu}{\rho}\right) \\
\left(q_{j}, \beta_{j}\right)_{1, m^{\prime}}\left(\frac{1}{\rho}(\alpha+(\rho(n-\gamma+1)-1) k), \frac{\nu}{\rho}\right)
\end{array} \mid \lambda s^{\frac{\nu}{k}}\right]\right) .
$$

Using (1.12), we can write the above equation as

$$
P \equiv\left(\frac{k}{\rho}\right)^{n-\gamma} \sum_{r=0}^{\infty} \frac{\prod_{i=1}^{n} \Gamma_{k}\left(p_{i}+\alpha_{i} r\right) \Gamma_{k}\left(\frac{1}{\rho}(\alpha+(\rho-1) k)+\frac{\nu}{\rho} r\right)}{\prod_{j=1}^{m} \Gamma_{k}\left(q_{j}+\beta_{j} r\right) \Gamma_{k}\left(\frac{1}{\rho}(\alpha+(\rho(n-\gamma+1)-1) k)+\frac{\nu}{\rho} r\right)} \frac{(\lambda)^{r}}{r !}\left(s^{1-\rho} \frac{d}{d s}\right)^{n}\left(s^{\frac{\alpha}{k}+\frac{\nu}{k}+\rho(n-\gamma)-1}\right) .
$$

Also, the above equation can be written as

$$
\begin{aligned}
P \equiv k^{n-\gamma} \rho^{\gamma} \sum_{r=0}^{\infty} \frac{\prod_{i=1}^{n} \Gamma_{k}\left(p_{i}+\alpha_{i} r\right) \Gamma_{k}\left(\frac{1}{\rho}(\alpha+(\rho-1) k)+\frac{\nu}{\rho} r\right)}{\prod_{j=1}^{m} \Gamma_{k}\left(q_{j}+\beta_{j} r\right) \Gamma_{k}\left(\frac{1}{\rho}(\alpha+(\rho(n-\gamma+1)-1) k)+\frac{\nu}{\rho} r\right)} \frac{(\lambda)^{r}}{r !} \\
\times \frac{\Gamma\left(\frac{1}{\rho}\left(\frac{\alpha}{k}+\frac{\nu r}{k}+(n-\gamma) \rho+\rho-1\right)\right.}{\Gamma\left(\frac{1}{\rho}\left(\frac{\alpha}{k}+\frac{\nu r}{k}-\gamma \rho+\rho-1\right)\right.} s^{\frac{\alpha}{k}+\frac{\nu}{k}-\rho \gamma-1} .
\end{aligned}
$$

Using (1.8), we obtain

$$
P \equiv\left(\frac{k}{\rho}\right)^{-\gamma} s^{\frac{\alpha}{k}-\rho \gamma-1}{ }_{n+1} \Phi_{m+1}^{k}\left[\begin{array}{c}
\left(p_{i}, \alpha_{i}\right)_{1, n^{\prime}}\left(\frac{1}{\rho}(\alpha+(\rho-1) k), \frac{\nu}{\rho}\right) \\
\left(q_{j}, \beta_{j}\right)_{1, m^{\prime}}\left(\frac{1}{\rho}(\alpha+(\rho(1-\gamma)-1) k), \frac{\nu}{\rho}\right)
\end{array} \mid \lambda s^{\frac{\nu}{k}}\right] .
$$

Theorem 4.2. Let $\gamma, \alpha \in \mathbb{C}$ such that $\mathfrak{R}(\gamma)>0, \mathfrak{R}(\alpha)>1+[\mathfrak{R}(\gamma)]-\mathfrak{R}(\gamma) ; \lambda \in \mathbb{C}, \rho>0, \nu>$ 0 , then for $\Delta>-1$, the Katugampola fractional differentiation ${ }_{\rho} D_{-}^{\gamma}$ for generalized $k$-Wright function ${ }_{n} \Phi_{m}^{k}(z)$ is given as

$$
\begin{aligned}
& \left(\rho _ { \rho } D _ { - } ^ { \gamma } \left(\tau ^ { - \frac { \alpha } { k } } { } _ { n } \Phi _ { m } ^ { k } \left[\begin{array}{c}
\left(p_{i}, \alpha_{i}\right)_{1, n} \\
\left.\left.\left.\left(q_{j}, \beta_{j}\right)_{1, m} \mid \lambda \tau^{-\frac{\nu}{k}}\right]\right)\right)(s)
\end{array}\right.\right.\right. \\
& \quad=\left(\frac{k}{\rho}\right)^{-\gamma} s^{-\rho \gamma-\frac{\alpha}{k}}{ }_{n+1} \Phi_{m+1}^{k}\left[\begin{array}{c}
\left(p_{i}, \alpha_{i}\right)_{1, n^{\prime}}\left(\frac{\alpha}{\rho}+k \gamma, \frac{\nu}{\rho}\right) \\
\left(q_{j}, \beta_{j}\right)_{1, m},\left(\frac{\alpha}{\rho}, \frac{\nu}{\rho}\right)
\end{array} \mid \lambda s^{-\frac{\nu}{k}}\right]
\end{aligned}
$$

Proof. According to Lemma 1.1, a generalized $k$-Wright function in both sides of the equation (4.2) exists for $s>0$. Let $n=1+[\Re(\gamma)]$. Then, we consider that

$$
Q \equiv\left({ }_{\rho} D_{-}^{\gamma}\left(\tau^{-\frac{\alpha}{k}}{ }_{n} \Phi_{m}^{k}\left[\begin{array}{c}
\left(p_{i}, \alpha_{i}\right)_{1, n} \\
\left(q_{j}, \beta_{j}\right)_{1, m}
\end{array} \mid \lambda \tau^{-\frac{\nu}{k}}\right]\right)\right)(s) .
$$

Using (1.4), we have

$$
Q \equiv\left(-s^{1-\rho} \frac{d}{d s}\right)^{n}\left(\rho_{-}^{n-\gamma}\left(\tau^{-\frac{\alpha}{k}}{ }_{n} \Phi_{m}^{k}\left[\begin{array}{l}
\left(p_{i}, \alpha_{i}\right)_{1, n} \mid \lambda \tau^{-\frac{\nu}{k}} \\
\left(q_{j}, \beta_{j}\right)_{1, m}
\end{array}\right]\right)\right)(s) .
$$


Using Theorem 3.2, we obtain

$$
Q \equiv\left(-s^{1-\rho} \frac{d}{d s}\right)^{n}\left(\frac{k}{\rho}\right)^{n-\gamma} s^{\rho(n-\gamma)-\frac{\alpha}{k}}{ }_{n+1} \Phi_{m+1}^{k}\left[\begin{array}{c}
\left(p_{i}, \alpha_{i}\right)_{1, n},\left(\frac{\alpha}{\rho}-k(n-\gamma), \frac{\nu}{\rho}\right) \\
\left(q_{j}, \beta_{j}\right)_{1, m},\left(\frac{\alpha}{\rho}, \frac{\nu}{\rho}\right)
\end{array} \mid \lambda s^{-\frac{\nu}{k}}\right] .
$$

Using (1.12), we can write the above equation as

$$
Q \equiv(-1)^{n}\left(\frac{k}{\rho}\right)^{n-\gamma} \sum_{r=0}^{\infty} \frac{\prod_{i=1}^{n} \Gamma_{k}\left(p_{i}+\alpha_{i} r\right) \Gamma_{k}\left(\frac{\alpha}{\rho}-(n-\gamma) k+\frac{\nu}{\rho} r\right)}{\prod_{j=1}^{m} \Gamma_{k}\left(q_{j}+\beta_{j} r\right) \Gamma_{k}\left(\frac{\alpha}{\rho}+\frac{\nu}{\rho} r\right)} \frac{(\lambda)^{r}}{r !}\left(s^{1-\rho} \frac{d}{d s}\right)^{n}\left(s^{\rho(n-\gamma)-\frac{\alpha}{k}-\frac{\nu}{k}}\right) .
$$

On simplifying the above equation, we obtain

$$
\begin{aligned}
Q \equiv(-1)^{n} k^{n-\gamma} \rho^{\gamma} \sum_{r=0}^{\infty} \frac{\prod_{i=1}^{n} \Gamma_{k}\left(p_{i}+\alpha_{i} r\right) \Gamma_{k}\left(\frac{\alpha}{\rho}-(n-\gamma) k+\frac{\nu}{\rho} r\right)}{\prod_{j=1}^{m} \Gamma_{k}\left(q_{j}+\beta_{j} r\right) \Gamma_{k}\left(\frac{\alpha}{\rho}+\frac{\nu}{\rho} r\right)} \frac{(\lambda)^{r}}{r !} \\
\quad \times \frac{\Gamma\left(1+(n-\gamma)-\frac{\alpha}{\rho k}-\frac{\nu}{\rho k} r\right)}{\Gamma\left(1-\gamma-\frac{\alpha}{\rho k}-\frac{\nu}{\rho k} r\right)}\left(s^{-\rho \gamma-\frac{\alpha}{k}-\frac{\nu}{k}}\right) .
\end{aligned}
$$

Using (1.8), we obtain

$$
\begin{aligned}
& Q \equiv(-1)^{n} \rho^{\gamma} \sum_{r=0}^{\infty} \frac{\prod_{i=1}^{n} \Gamma_{k}\left(p_{i}+\alpha_{i} r\right)}{\prod_{j=1}^{m} \Gamma_{k}\left(q_{j}+\beta_{j} r\right) \Gamma\left(\frac{\alpha}{\rho k}+\frac{\nu}{\rho k} r\right)} \frac{(\lambda)^{r}}{r !} \\
& \times \frac{\Gamma\left(\gamma-n+\frac{\alpha}{\rho k}+\frac{\nu}{\rho k} r\right) \Gamma\left(1-\left(\gamma-n+\frac{\alpha}{\rho k}+\frac{\nu}{\rho k} r\right)\right)}{\Gamma\left(1-\left(\gamma+\frac{\alpha}{\rho k}+\frac{\nu}{\rho k} r\right)\right)}\left(s^{-\rho \gamma-\frac{\alpha}{k}-\frac{\nu}{k}}\right) .
\end{aligned}
$$

Using (1.9), we have

$$
\begin{aligned}
\Gamma\left(\gamma-n+\frac{\alpha}{\rho k}+\frac{\nu}{\rho k} r\right) & \Gamma\left(1-\left(\gamma-n+\frac{\alpha}{\rho k}+\frac{\nu}{\rho k} r\right)\right) \\
& =\frac{\pi}{\sin \left[\left(\gamma+\frac{\alpha}{\rho k}+\frac{\nu}{\rho k} r\right) \pi-n \pi\right]} \\
& =\frac{\pi}{\sin \left[\left(\gamma+\frac{\alpha}{\rho k}+\frac{\nu}{\rho k} r\right) \pi\right] \cos (n \pi)} \\
& =\frac{(-1)^{n} \pi}{\sin \left[\left(\gamma+\frac{\alpha}{\rho k}+\frac{\nu}{\rho k} r\right) \pi\right]}
\end{aligned}
$$

and

$$
\frac{1}{\Gamma\left(1-\left(\gamma+\frac{\alpha}{\rho k}+\frac{\nu}{\rho k} r\right)\right)}=\frac{\Gamma\left(\gamma+\frac{\alpha}{\rho k}+\frac{\nu}{\rho k} r\right) \sin \left[\left(\gamma+\frac{\alpha}{\rho k}+\frac{\nu}{\rho k} r\right) \pi\right]}{\pi} .
$$

Substituting (4.4) and (4.5) in (4.3) and finally by using (1.8), we obtain

$$
Q \equiv\left(\frac{k}{\rho}\right)^{-\gamma} s^{-\rho \gamma-\frac{\alpha}{k}}{ }_{n+1} \Phi_{m+1}^{k}\left[\begin{array}{c}
\left(p_{i}, \alpha_{i}\right)_{1, n},\left(\frac{\alpha}{\rho}+k \gamma, \frac{\nu}{\rho}\right) \\
\left(q_{j}, \beta_{j}\right)_{1, m},\left(\frac{\alpha}{\rho}, \frac{\nu}{\rho}\right)
\end{array} \mid \lambda s^{-\frac{\nu}{k}}\right]
$$




\section{Concluding Remarks}

- If $\rho=1$, then

Theorems 3.1, 3.2, 4.1 and 4.2, are reduced to Theorems 2, 3, 4 and 5 respectively (see [4]).

- Some general properties of the Katugampola fractional integrals and derivatives for the power function $\varphi(s)=s^{\alpha-1}$ and the exponential function $e^{-\lambda s^{\rho}}$ are investigated.

- The Katugampola fractional integration $\rho_{0+}^{\gamma}$ and $\rho_{\rho} I_{-}^{\gamma}$ for generalized $k$-Wright function ${ }_{n} \Phi_{m}^{k}(z)$ are established.

- The Katugampola fractional differentiation ${ }_{\rho} D_{0+}^{\gamma}$ and ${ }_{\rho} D_{-}^{\gamma}$ for generalized $k-$ Wright function ${ }_{n} \Phi_{m}^{k}(z)$ are established.

\section{ACKNOWLEDGMENT}

The authors are would like to thank the reviewers for their important remarks and suggestions.

\section{RefEREnCES}

[1] R. Almeida, A.B. Malinowska, T. Odzijewicz, Fractional differential equations with dependence on the Caputo?Katugampola derivative, J. Comput. Nonlinear Dynam. 11 (2016) 061017. https://doi.org/10.1115/1. 4034432.

[2] R. Diaz, E. Pariguan, On hypergeometric functions and Pochhammer k-symbol. Divulgaciones Math. 15 (2) (2007) 179-192.

[3] K.S. Gehlot, J.C. Prajapati, On generalization of K-Wright function and its properties, Pac. J. Appl. Math. 5 (2) (2013) 81-88.

[4] K.S. Gehlot, J.C. Prajapati, Fractional calculus of generalized K-Wright function, J. Fraction. Calc. Appl. 4 (2) (2013) 83-289.

[5] U.N. Katugampola, New approach to a generalized fractional integral, Appl. Math. Comput. 218 (2011) 860-865. https://doi.org/10.1016/j.amc.2011.03.062.

[6] U.N. Katugampola, A new approach to generalized fractional derivatives, Bull. Math. Anal. Appl. 6 (4), (2014), 1-15.

[7] U.N. Katugampola, Existence and uniqueness results for a class of generalized fractional differential equations, arXiv:1411.5229v2[math.CA] 9 Jun (2014).

[8] A.A. Kilbas, H.M. Srivastava, J.J. Trujillo, Theory and Applications of Fractional Differential Equations, Elsevier, Amsterdam (2006).

[9] D.S. Oliveira, E.C. De Oliveira, Hilfer-Katugampola fractional derivative, Comput. Appl. Math. 37 (2018), 3672-3690. https://doi.org/10.1007/s40314-017-0536-8.

[10] A.Y.A. Salamooni, D.D. Pawar, Unique positive solution for nonlinear Caputo-type fractional q-difference equations with nonlocal and Stieltjes integral boundary conditions, Fraction. Differ. Calc. 9 (2) (2019), 295-307.

[11] A.Y.A. Salamooni, D.D. Pawar, Existence and uniqueness of generalised fractional Cauchy-type problem, Univ. J. Math. Appl. 3 (3) (2020), 121-128.

[12] A.Y.A. Salamooni, D.D. Pawar, Existence and uniqueness of boundary value problems for Hilfer-Hadamard-type fractional differential equations, Ganita, 70 (2) (2020), 01-16. 
[13] A.Y.A. Salamooni, D.D. Pawar, Existence and stability results for Hilfer-Katugampola-type fractional implicit differential equations with nonlocal conditions, J. Nonlinear Sci. Appl. 14 (3) (2021), 124-138. http://dx. doi.org/ 10.22436/jnsa.014.03.02.

[14] A.Y.A. Salamooni, D.D. Pawar, Existence and uniqueness of nonlocal boundary conditions for Hilfer-Hadamardtype fractional differential equations, Adv. Differ. Equations, 2021 (2021), 198. https://doi.org/10.1186/ s13662-021-03358-0.

[15] S.G. Samko, A.A. Kilbas, O.I. Marichev, Fractional integrals and derivatives: theory and applications, Gordon and Breach, New York (1993). 